\title{
Rapid distortion theory for compressible homogeneous turbulence under isotropic mean strain
}

\author{
G. A. Blaisdell \\ School of Aeronautics and Astronautics, Purdue University, West Lafayette, Indiana 47907-1282 \\ G. N. Coleman \\ Department of Mechanical and Aerospace Engineering, University of California, Los Angeles, \\ California 90024-1597
}

N. N. Mansour

NASA Ames Research Center, Moffett Field, California 94035

(Received 18 August 1995; accepted 30 May 1996)

\begin{abstract}
Isotropic compressible turbulence subjected to rapid isotropic compression is studied using inviscid rapid distortion theory (RDT) and direct numerical simulation. An exact solution to the rapid distortion problem is given. Comparisons are made between the simulation results and the RDT solution, as well as previously studied limiting cases of the RDT solution. The comparisons illustrate the range of applicability of the RDT solutions. Implications for the use of RDT results in modeling compressible turbulent flows are briefly discussed. (C) 1996 American Institute of Physics. [S1070-6631(96)03209-6]
\end{abstract}

\section{INTRODUCTION}

Compressibility effects on turbulence are important in many engineering applications and have been the subject of numerous recent investigations. In order to accurately model compressible turbulent flows there is a need to improve current turbulence models and to increase our understanding of compressible turbulence. One tool that has proven useful in turbulence model development and in gaining insight into turbulent flows is rapid distortion theory (RDT). Within RDT the time scale of the mean flow is assumed much shorter than that of the turbulence so that nonlinear interactions within the turbulence can be neglected. In the current investigation we consider the rapid distortion problem for isotropic turbulence subjected to isotropic mean compression.

Isotropic mean compression is considered for two reasons. First, the problem is tractable and, as shown in section III, an exact solution to the inviscid RDT problem exists. The second reason is that many turbulence models are formulated in terms of an isotropic part and an anisotropic part. For such models, the case of isotropic mean compression would provide information needed to determine the model constants.

In order to check the validity of the RDT solution and the relevance of the assumptions made, the RDT results are compared to those of direct numerical simulations (DNS). We find that the exact RDT solution is of limited utility. In spite of the limitations, the analysis does yield insight into compressible turbulence and brings out some issues that need to be considered in modeling compressible turbulent flows.

The RDT problem for compressible homogeneous turbulence under mean strain has been considered previously by Sabelnikov, ${ }^{1}$ Durbin and Zeman, ${ }^{2}$ Jacquin et al. ${ }^{3}$ and Cambon et al. ${ }^{4}$ Sabelnikov considered a constant strain rate, which is inconsistent with the mean momentum equation under the restrictions of homogeneous turbulence. The flow obtained with a constant strain rate may be quasi- homogeneous in the sense discussed in Ref. 2; however, since the flow is not strictly homogeneous, the results are not compared to those of the current study. Durbin and Zeman considered anisotropic strains and found approximate solutions to the RDT equations by using the WKB (Wentzel, Kramers, Brillouin) method (see e.g., Bender and Orszag ${ }^{5}$ ) and assuming high frequencies and low turbulent Mach numbers. Jacquin et al. $^{3}$ and Cambon et al. $^{4}$ also considered anisotropic strains. They found solutions to the RDT problem under the "pressure released" approximation, which corresponds to the limit of very large strain rates but does not require the low Mach number approximation used by Durbin and Zeman. ${ }^{2}$ Cambon et al. ${ }^{4}$ also presented solutions in the limit of high frequencies and low turbulent Mach numbers and performed DNS spanning the range from the highfrequency limit to the pressure-released limit. In the current paper, we find exact solutions to the RDT problem for the special case of isotropic mean strain without the need for any of the above approximations, other than that of rapid strain rate.

Previous simulations of compressible turbulence under isotropic mean strain have been done by Coleman and Mansour. ${ }^{6,7}$ In Ref. 6 a simulation was performed at a very low turbulent Mach number $\left(M_{T}=0.04\right)$. The effect of the variation of the kinematic viscosity due to the mean flow compression was examined, but no other compressibility effects were considered. In Ref. 7 the simulations were at higher turbulent Mach numbers. Comparisons were made with turbulence models for compressibility terms arising in the turbulent kinetic energy equation (see section II below), but satisfactory agreement was not obtained.

The paper is organized as follows. In section II the equations for the turbulent fields are simplified under the assumptions of RDT. In section III a simple exact solution to the RDT problem is given for the case of a monatomic gas ( $\gamma=5 / 3$, where $\gamma$ is the ratio of specific heats). A solution for general values of $\gamma$ is given in the appendix, but is not 


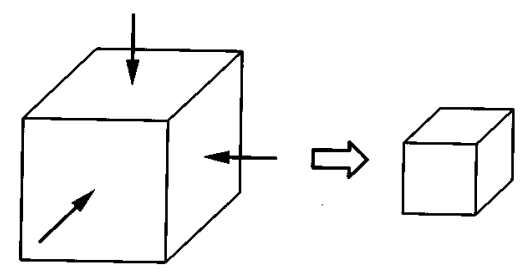

FIG. 1. Schematic of isotropic compression.

used because of its complexity. Limiting cases of the RDT solution for high frequencies and high strain rates are then considered. The RDT predictions are compared to DNS results in section IV, and the validity of the RDT assumptions are discussed. Finally, conclusions are made in section V.

\section{GOVERNING EQUATIONS}

Restrictions on the mean flow required for compressible turbulence to remain homogeneous have been given by Blaisdell et al. ${ }^{8}$ and Cambon et ll $^{4}$ They have also been presented for the case of strained flows by Durbin and Zeman $^{2}$ who considered the RDT problem for anisotropic mean strain. For isotropic mean strain the mean velocity gradient tensor has the form

$$
\frac{\partial \widetilde{u_{i}}}{\partial x_{j}}=S(t) \delta_{i j}=\frac{S_{0}}{\left(1+S_{0} t\right)} \delta_{i j},
$$

where $S_{0}$ is the initial mean strain rate $\left(S_{0}<0\right.$ for compression). The tilde over the velocity indicates a Favre or massweighted average. The effect of the isotropic compression upon a fluid element is depicted in Fig. 1. As a consequence of the change in volume, the mean density is given by

$$
\bar{\rho}(t)=\frac{\bar{\rho}(0)}{\left(1+S_{0} t\right)^{3}} .
$$

Note that for the case of a compressive strain there is a singularity at $\left|S_{0}\right| t=1$. This corresponds to the time when all space is compressed to zero volume. In the discussion below, results are presented for time in the range $0 \leqslant\left|S_{0}\right| t \leqslant 0.8$.

The assumptions for RDT are discussed in detail by Hunt and Carruthers. ${ }^{9}$ RDT is a linearized theory in which the time scale of the mean flow is assumed much smaller than the time scale of the turbulence at a given length scale. Based on this assumption, the nonlinear terms in the equations of motion can be neglected relative to the terms containing the mean deformation rate. For the large energy containing eddies the time scale of the turbulence is longer than that of the small dissipation scales. Therefore, a larger mean deformation rate is needed to justify RDT for the small scales than for the large scales. Also, RDT is valid for short nondimensional times even when the mean deformation rate is not large. Thus for large but finite deformation rates, RDT will be valid initially but become less accurate at later nondimensional times. These effects are seen in the comparisons made below. Because they are linear, the viscous terms could be included in the formulation. Instead, we assume the vis- cous decay time is long compared to the time scale of the mean flow, and use inviscid RDT, which substantially simplifies the analysis.

Since the nonlinear, viscous and heat conduction terms are neglected, the mean flow and the fluctuations follow the isentropic relations. Specifically, the mean pressure is related to the mean density and is given by

$$
\bar{p}(t)=\frac{\bar{p}(0)}{\left(1+S_{0} t\right)^{3 \gamma}},
$$

where $\gamma$ is the ratio of specific heats. The linearized, inviscid equations for the fluctuating quantities are

$$
\begin{aligned}
& \frac{\bar{D} u_{i}^{\prime}}{D t}=-S(t) u_{i}^{\prime}-\frac{1}{\bar{\rho}(t)} \frac{\partial p^{\prime}}{\partial x_{i}}, \\
& \frac{\bar{D} p^{\prime}}{D t}=-\gamma \bar{p}(t) d^{\prime}-3 \gamma S(t) p^{\prime}, \\
& \frac{\bar{D} s^{\prime}}{D t}=0,
\end{aligned}
$$

where

$$
\frac{\bar{D}}{D t}=\frac{\partial}{\partial t}+S(t) x_{k} \frac{\partial}{\partial x_{k}}
$$

is the substantial derivative based on the mean velocity, primed quantities denote fluctuations, and $s$ is the entropy. (The fluctuating density and temperature are related to $p^{\prime}$ through the isentropic relations, and therefore only an equation for the pressure is needed.)

Splitting the problem into acoustic and vortical modes yields considerable insight, as is discussed below. Taking the divergence and the curl of the fluctuating momentum equation (4) gives

$$
\begin{aligned}
& \frac{\bar{D} d^{\prime}}{D t}=-2 S(t) d^{\prime}-\frac{1}{\bar{\rho}} \nabla^{2} p^{\prime}, \\
& \frac{\bar{D} \omega_{i}^{\prime}}{D t}=-2 S(t) \omega_{i}^{\prime},
\end{aligned}
$$

where $d^{\prime}$ is the dilatation (fluctuating divergence of velocity) and $\omega_{i}^{\prime}$ is the fluctuating vorticity.

In order to be able to use periodic boundary conditions in the DNS and Fourier analysis for the RDT, a transformation is employed ${ }^{10}$ in which the coordinate system deforms with the mean flow, such that

$$
x_{i}^{\prime}=B_{i j}(t) x_{j},
$$

where

$$
B_{i j}(t)=B(t) \delta_{i j}=\frac{1}{\left(1+S_{0} t\right)} \delta_{i j}
$$

is the coordinate transformation matrix. The resulting computational domain for the DNS deforms as shown in Fig. 1.

The equations for the fluctuations are most easily solved in Fourier space. After transformation, they are 


$$
\begin{aligned}
& \frac{d \hat{p}}{d t}=-\gamma \bar{p}(t) \hat{d}-3 \gamma S(t) \hat{p}, \\
& \frac{d \hat{d}}{d t}=-2 S(t) \hat{d}+\frac{B^{2}(t)}{\bar{\rho}(t)} \kappa^{2} \hat{p}, \\
& \frac{d \hat{\omega}_{i}}{d t}=-2 S(t) \hat{\omega}_{i},
\end{aligned}
$$

where $\wedge$ denotes a Fourier coefficient, and $\kappa$ is the magnitude of the wavenumber vector, $\vec{\kappa}$. (The entropy equation does not enter into the formulation since it reduces to $s^{\prime}=$ constant.) One point that is clear from these equations is that the vorticity mode and acoustic mode are decoupled. This is not true for anisotropic mean strains, in which case the vorticity acts as a source term in the dilatation equation. ${ }^{2,4}$ This decoupling has implications for turbulence modeling and is discussed further below.

There are two additional terms due to compressibility that enter into the two-equation turbulence modeling problem for homogeneous turbulence. They appear in the equation for turbulent kinetic energy, which is

$$
\frac{\bar{D} k}{D t}=\mathscr{P}-\varepsilon_{s}-\varepsilon_{d}+\Pi_{d},
$$

where $k=\overline{\rho u_{i}^{\prime} u_{i}^{\prime}} / 2$ is the turbulent kinetic energy per unit volume, $\mathscr{P}=\overline{\rho u_{i}^{\prime} u_{j}^{\prime}} \widetilde{u}_{i, j}$ is the production term, $\varepsilon_{s}=\bar{\mu} \overline{\omega_{i}^{\prime} \omega_{i}^{\prime}}$ is the solenoidal dissipation rate, $\varepsilon_{d}=(4 / 3) \tilde{\mu} \overline{d^{\prime} d^{\prime}}$ is the dilatational dissipation rate, and $\Pi_{d}=\overline{p^{\prime} d^{\prime}}$ is the pressuredilatation correlation. The solenoidal dissipation rate, $\varepsilon_{s}$, is the same as the dissipation rate in incompressible homogeneous turbulence and is typically modeled using the usual incompressible dissipation rate equation as follows:

$$
\frac{\bar{D} \varepsilon_{s}}{D t}=C_{\varepsilon 1} \mathscr{P} \frac{\varepsilon_{s}}{k}-C_{\varepsilon 2} \frac{\varepsilon_{s}^{2}}{k} .
$$

The dilatational dissipation rate and the pressure-dilatation correlation are the two additional compressibility terms. The dilatational dissipation represents the rate of dissipation of turbulent kinetic energy due to the divergence of velocity, and is an irreversible process. On the other hand, the pressure-dilatation correlation represents the reversible transfer of energy from kinetic to internal energy. One of the goals of this investigation is to use rapid distortion theory to gain more insight into these two terms.

A consequence of the decoupling of the vorticity and acoustic modes described above is that the ratio of the dilatational to the solenoidal dissipation rate is highly dependent on initial conditions. This was shown by Coleman and Mansour, ${ }^{7}$ who performed DNS of compressible turbulence undergoing isotropic strain at intermediate values of the strain rate. However, the turbulence models for the dilatational dissipation rate suggested by Zeman ${ }^{11,12}$ and Sarkar ${ }^{13,14}$ are not sensitive to initial conditions. These models are of the form $\varepsilon_{d} / \varepsilon_{s}=F\left(M_{T}\right)$, where $M_{T}$ is the turbulent Mach number defined by $M_{T}^{2}=\frac{T}{u_{i}^{2} u_{i}^{2}} / c^{2}$ and $c^{2}=\gamma \bar{p} / \bar{\rho}$. Such an explicit algebraic relation is insensitive to the initial value of this ratio and, therefore, in general such models cannot be expected to give accurate results for flows subject to this type of deformation. This point will be addressed further below, as the behavior of the dilatational dissipation and pressure-dilatation correlation are investigated.

\section{RDT SOLUTION}

Equations (12)-(14), which describe the evolution of the turbulence under RDT, can be solved exactly, although the acoustic mode solution is greatly simplified by assuming a monatomic gas. We begin by considering the vorticity mode.

\section{A. Vorticity mode}

The differential equation for the vorticity (14) is the same as that for incompressible flow, and it can easily be solved since it is separable. Using the resulting solution to form the enstrophy, one has

$$
\overline{\omega_{i} \omega_{i}}(t)=\frac{\overline{\omega_{i} \omega_{i}}(0)}{\left(1+S_{0} t\right)^{4}} .
$$

The evolution of the solenoidal dissipation rate, $\varepsilon_{s}$, can be found from the above formula for the enstrophy and the known history of the viscosity. (In the current simulations the viscosity is held fixed. However, if the viscosity is temperature dependent, it can easily be found from the mean temperature, which is related to the mean density and pressure.) The effect of changes in mean viscosity on the dissipation rate has been considered in Ref. 7. In the current work we concentrate on $\varepsilon_{d}$ and $\Pi_{d}$, the two added compressibility terms in the turbulent kinetic energy equation.

In order to aid the discussion below it is useful to decompose the velocity field into solenoidal and dilatational parts using Helmholtz's decomposition (see, for example Ref. 15). The solenoidal velocity, $u_{i}^{s^{\prime}}$, is divergence free and is determined by the vorticity field; the dilatational velocity, $u_{i}^{d^{\prime}}$, is irrotational and is determined by the dilatation. In Fourier space the solenoidal velocity is given by

$$
\hat{u}_{\ell}^{s^{\prime}}=\left(1+S_{0} t\right) \frac{i \epsilon_{\ell m n} \kappa_{m}}{\kappa^{2}} \hat{\omega}_{n},
$$

and its variance is

$$
\overline{u_{\ell}^{s^{\prime}} u_{\ell}^{s^{\prime}}}(t)=\frac{\overline{u_{\ell}^{s^{\prime}} u_{\ell}^{s^{\prime}}}(0)}{\left(1+S_{0} t\right)^{2}} .
$$

The dilatational velocity is part of the acoustic mode, which is discussed next.

\section{B. Acoustic mode exact solution}

The acoustic mode is more difficult to analyze because it involves two coupled equations. The equations can be decoupled by using integrating factors. We let $\hat{d}=I(t) \breve{d}$ $=\breve{d} /\left(1+S_{0} t\right)^{2}$ and $\hat{p}=J(t) \breve{p}=\breve{p} /\left(1+S_{0} t\right)^{3 \gamma}$, and then combine to form a second order equation. We choose to solve for $\breve{d}$ and then use equation (13) to obtain $\hat{p}$ from $\hat{d}$. The resulting equation for $\breve{d}$ is

$$
\frac{d}{d t}\left[\left(1+S_{0} t\right)^{3(\gamma-1)} \frac{d}{d t} \breve{d}\right]=-\frac{c_{0}^{2} \kappa^{2}}{\left(1+S_{0} t\right)^{2}} \breve{d},
$$


where $c_{0}^{2}=\gamma \bar{p}(0) / \bar{\rho}(0)$ is the square of the initial speed of sound based on mean properties. This differential equation can be solved for general values of $\gamma$ in terms of Bessel functions (see the Appendix), but it is greatly simplified by considering a monatomic gas $(\gamma=5 / 3)$. For this case the exponent $3(\gamma-1)=2$. Upon making a change of variables, $\tau=1 /\left(1+S_{0} t\right)$, one has

$$
\frac{d^{2}}{d \tau^{2}} \breve{d}+\left(\frac{c_{0} \kappa}{S_{0}}\right)^{2} \breve{d}=0
$$

which is easily solved in terms of $\sin \left(c_{0} \kappa \tau / S_{0}\right)$ and $\cos \left(c_{0} \kappa \tau / S_{0}\right)$. The resulting solution for the Fourier coefficients for the pressure and dilatation are

$$
\begin{aligned}
\hat{p}(\vec{\kappa}, t)= & \frac{\hat{p}(\vec{\kappa}, 0)}{\left(1+S_{0} t\right)^{5}} \cos \left(\frac{c_{0} \kappa t}{1+S_{0} t}\right) \\
& -\frac{\rho_{0} c_{0} \hat{d}(\vec{\kappa}, 0)}{\kappa\left(1+S_{0} t\right)^{5}} \sin \left(\frac{c_{0} \kappa t}{1+S_{0} t}\right)
\end{aligned}
$$

and

$$
\begin{aligned}
\hat{d}(\vec{\kappa}, t)= & \frac{\hat{d}(\vec{\kappa}, 0)}{\left(1+S_{0} t\right)^{2}} \cos \left(\frac{c_{0} \kappa t}{1+S_{0} t}\right) \\
& +\frac{\kappa \hat{p}(\vec{\kappa}, 0)}{\rho_{0} c_{0}\left(1+S_{0} t\right)^{2}} \sin \left(\frac{c_{0} \kappa t}{1+S_{0} t}\right),
\end{aligned}
$$

where $\rho_{0}$ has been used to denote $\bar{\rho}(0)$. This solution is the same as the approximate WKB solution found by Durbin and Zeman, ${ }^{2}$ except that here we see that for the case of isotropic strain and a monatomic gas $(\gamma=5 / 3)$ it is exact and the highfrequency assumption made by Durbin and Zeman is unnecessary.

The turbulence statistics of interest can be found by integrating the Fourier coefficients. First define the threedimensional spectra

$$
\begin{aligned}
& E_{d d}(\kappa, t)=\int_{0}^{\pi} \int_{0}^{2 \pi} \hat{d}(\vec{\kappa}, t) \hat{d}^{*}(\vec{\kappa}, t) \kappa^{2} \sin \theta d \phi d \theta, \\
& E_{p p}(\kappa, t)=\int_{0}^{\pi} \int_{0}^{2 \pi} \hat{p}(\vec{\kappa}, t) \hat{p}^{*}(\vec{\kappa}, t) \kappa^{2} \sin \theta d \phi d \theta, \\
& E_{p d}(\kappa, t)=\int_{0}^{\pi} \int_{0}^{2 \pi} \hat{p}(\vec{\kappa}, t) \hat{d}^{*}(\vec{\kappa}, t) \kappa^{2} \sin \theta d \phi d \theta, \\
& E_{d p}(\kappa, t)=\int_{0}^{\pi} \int_{0}^{2 \pi} \hat{d}(\vec{\kappa}, t) \hat{p}^{*}(\vec{\kappa}, t) \kappa^{2} \sin \theta d \phi d \theta,
\end{aligned}
$$

where ${ }^{*}$ denotes the complex conjugate. The temporal evolution of the dilatation is then given in terms of the initial spectra,

$$
\begin{aligned}
\overline{d^{\prime} d^{\prime}}(t)= & \int_{0}^{\infty} E_{d d}(\kappa, t) d \kappa=\frac{1}{2\left(1+S_{0} t\right)^{4}}\left\{2 \overline{d^{\prime} d^{\prime}}(0)\right. \\
& +\int_{0}^{\infty}\left[\left(\frac{\kappa}{\rho_{o} c_{0}}\right)^{2} E_{p p}(\kappa, 0)-E_{d d}(\kappa, 0)\right] \\
& \times\left[1-\cos \left(\frac{2 c_{0} \kappa t}{1+S_{0} t}\right)\right] d \kappa+\int_{0}^{\infty} \frac{\kappa}{\rho_{0} c_{0}}\left(E_{p d}(\kappa, 0)\right. \\
& \left.\left.+E_{d p}(\kappa, 0)\right) \sin \left(\frac{2 c_{0} \kappa t}{1+S_{0} t}\right) d \kappa\right\} .
\end{aligned}
$$

The pressure-dilatation correlation is found from

$$
\begin{aligned}
\overline{p^{\prime} d^{\prime}}(t)= & \frac{1}{2} \int_{0}^{\infty}\left(E_{p d}(\kappa, t)+E_{d p}(\kappa, t)\right) d \kappa \\
= & \frac{1}{2\left(1+S_{0} t\right)^{7}}\left\{\int _ { 0 } ^ { \infty } \left(E_{p d}(\kappa, 0)\right.\right. \\
& \left.+E_{d p}(\kappa, 0)\right) \cos \left(\frac{2 c_{0} \kappa t}{1+S_{0} t}\right) d \kappa \\
& +\int_{0}^{\infty}\left[\frac{\kappa}{\rho_{0} c_{0}} E_{p p}(\kappa, 0)\right. \\
& \left.\left.-\frac{\rho_{0} c_{0}}{\kappa} E_{d d}(\kappa, 0)\right] \sin \left(\frac{2 c_{0} \kappa t}{1+S_{0} t}\right) d \kappa\right\} .
\end{aligned}
$$

The pressure-dilatation correlation represents an energy exchange between the velocity and pressure fields, and the above solution shows how (i.e., in which direction) energy in the acoustic mode is transferred for a given set of initial conditions.

In order to complete the solution, the evolution of other related statistics is found. The pressure variance is determined by

$$
\begin{aligned}
\overline{p^{\prime} p^{\prime}}(t)= & \int_{0}^{\infty} E_{p p}(\kappa, t) d \kappa \\
= & \frac{1}{2\left(1+S_{0} t\right)^{10}}\left\{2 \overline{p^{\prime} p^{\prime}}(0)\right. \\
& +\int_{0}^{\infty}\left[\left(\frac{\rho_{0} c_{0}}{\kappa}\right)^{2} E_{d d}(\kappa, 0)-E_{p p}(\kappa, 0)\right] \\
& \times\left[1-\cos \left(\frac{2 c_{0} \kappa t}{1+S_{0} t}\right)\right] d \kappa-\int_{0}^{\infty} \frac{\rho_{0} c_{0}}{\kappa}\left(E_{p d}(\kappa, 0)\right. \\
& \left.\left.+E_{d p}(\kappa, 0)\right) \sin \left(\frac{2 c_{0} \kappa t}{1+S_{0} t}\right) d \kappa\right\} .
\end{aligned}
$$

The mean-square magnitude of the pressure gradient is given by 


$$
\begin{aligned}
\overline{\boldsymbol{\nabla} p^{\prime} \cdot \boldsymbol{\nabla} p^{\prime}}(t)= & \frac{1}{\left(1+S_{0} t\right)^{2}} \int_{0}^{\infty} \kappa^{2} E_{p p}(\kappa, t) d \kappa \\
= & \frac{1}{2\left(1+S_{0} t\right)^{12}}\left\{2 \overline{\nabla p^{\prime} \cdot \nabla p^{\prime}}(0)\right. \\
& +\int_{0}^{\infty}\left[\left(\rho_{0} c_{0}\right)^{2} E_{d d}(\kappa, 0)-\kappa^{2} E_{p p}(\kappa, 0)\right] \\
& \times\left[1-\cos \left(\frac{2 c_{0} \kappa t}{1+S_{0} t}\right)\right] d \kappa \\
& -\int_{0}^{\infty} \rho_{0} c_{0} \kappa\left(E_{p d}(\kappa, 0)\right. \\
& \left.\left.+E_{d p}(\kappa, 0)\right) \sin \left(\frac{2 c_{0} \kappa t}{1+S_{0} t}\right) d \kappa\right\}
\end{aligned}
$$

Last, the variance of the dilatational velocity is obtained from

$$
\begin{aligned}
\overline{u_{i}^{d^{\prime}} u_{i}^{d^{\prime}}}(t)= & \left(1+S_{0} t\right)^{2} \int_{0}^{\infty} \frac{E_{d d}(\kappa, t)}{\kappa^{2}} d \kappa \\
= & \frac{1}{2\left(1+S_{0} t\right)^{2}}\left\{2 \overline{u_{i}^{d^{\prime}} u_{i}^{d^{\prime}}}(0)\right. \\
& +\int_{0}^{\infty}\left[\left(\frac{1}{\rho_{0} c_{0}}\right)^{2} E_{p p}(\kappa, 0)-\frac{E_{d d}(\kappa, 0)}{\kappa^{2}}\right][1 \\
& \left.-\cos \left(\frac{2 c_{0} \kappa t}{1+S_{0} t}\right)\right] d \kappa-\int_{0}^{\infty} \frac{1}{\rho_{0} c_{0} \kappa}\left(E_{p d}(\kappa, 0)\right. \\
& \left.\left.+E_{d p}(\kappa, 0)\right) \sin \left(\frac{2 c_{0} \kappa t}{1+S_{0} t}\right) d \kappa\right\}
\end{aligned}
$$

The solutions given in Eqs. (28)-(32) are fairly complex and require knowledge of the initial spectra. Three physically significant cases will now be examined for which the solutions can be simplified.

\section{Acoustic equilibrium}

Sarkar et al. ${ }^{13}$ introduced the concept of acoustic equilibrium as applied to compressible turbulence in their study of decaying isotropic turbulence. For an acoustic field in equilibrium the velocity and pressure fields are in balance; there is no net energy exchange between them. Therefore, the pressure-dilatation correlation is zero. If the acoustic field is in equilibrium at all wavenumbers (rather than just in a global sense), then the pressure-dilatation spectrum will be zero,

$$
E_{p d}(\kappa, 0)+E_{d p}(\kappa, 0)=0,
$$

and the distribution of energy in the velocity and pressure fields will be balanced, as reflected by the following relation between their spectra

$$
E_{p p}(\kappa, 0)-\left(\frac{\rho_{0} c_{0}}{\kappa}\right)^{2} E_{d d}(\kappa, 0)=0 .
$$

[Note that Eqs. (33) and (34) hold at each $\kappa$.]
Sarkar et al. defined an acoustic equilibrium parameter, $F$, which can be obtained by integrating Eq. (34) over wavenumbers and rearranging to get

$$
F=\frac{\vec{\rho}^{2} c^{2} \overline{u_{i}^{d^{\prime}} u_{i}^{d^{\prime}}}}{\overline{p^{\prime} p^{\prime}}} .
$$

Acoustic equilibrium is attained if $F=1$. Equation (34) can also be multiplied by $\kappa^{2}$ and integrated to obtain an alternate acoustic equilibrium parameter defined by

$$
F^{\prime}=\frac{\overrightarrow{\rho^{2} c^{2} \overline{d^{\prime} d^{\prime}}}}{\overline{\nabla p^{\prime} \cdot \nabla p^{\prime}}}
$$

This parameter is more sensitive than $F$ to the small scales of the turbulence. However, the structure of the DNS code used in the current study makes calculation of $F$ computationally expensive while $F^{\prime}$ is relatively inexpensive to compute. It should be noted that the definition of $F$ given by Sarkar et al. is in terms of the compressible part of the pressure (see Ref. 13). However, it is not feasible to decompose the pressure field during the course of the DNS, and so here the full pressure is used instead. For flows with moderate to high magnitudes of the acoustic field there is little difference, while for flows with a weak acoustic field the equilibrium value of $F$ can be significantly smaller than 1 .

In addition to the pressure and velocity fields being balanced, as measured by the acoustic equilibrium parameter $F$ or $F^{\prime}$, it is necessary for the pressure-dilatation to be small in order for the acoustic field to be in equilibrium. If $\overline{p^{\prime} d^{\prime}}$ is large, then energy will be shifted between the pressure and velocity fields even though they may initially be in balance. Such a case is discussed in section IV.

If it is assumed that the initial flow field is in acoustic equilibrium, then the integrals in Eqs. (28)-(32) drop out leaving the following relations for the temporal evolution of the statistics:

$$
\begin{aligned}
& \overline{d^{\prime} d^{\prime}}(t)=\frac{\overline{d^{\prime} d^{\prime}}(0)}{\left(1+S_{0} t\right)^{4}}, \\
& \overline{p^{\prime} d^{\prime}}(t)=0, \\
& \overline{p^{\prime} p^{\prime}}(t)=\frac{\overline{p^{\prime} p^{\prime}}(0)}{\left(1+S_{0} t\right)^{10}}, \\
& \overline{\nabla p^{\prime} \cdot \nabla p^{\prime}}(t)=\frac{\overline{\nabla p^{\prime} \cdot \nabla p^{\prime}(0)}}{\left(1+S_{0} t\right)^{12}}, \\
& \overline{u_{i}^{d^{\prime}} u_{i}^{d^{\prime}}}(t)=\frac{\overline{u_{i}^{d^{\prime}} u_{i}^{d^{\prime}}}(0)}{\left(1+S_{0} t\right)^{2}} .
\end{aligned}
$$

When these solutions are substituted into the defining equations for $F$ and $F^{\prime}$ and the evolution of the mean density and speed of sound are accounted for, one sees that the flow field remains in acoustic equilibrium. The state of acoustic equilibrium or non-equilibrium is important to how the turbulence evolves, as will be demonstrated by the DNS results presented in section IV. 


\section{High frequency limit}

An approximation that can be applied to the solutions given in Eqs. (28) -(32) is the high frequency limit considered by Durbin and Zeman. ${ }^{2}$ (Hereafter referred to as HF RDT.) If $S_{0} /\left(c_{0} \kappa\right) \ll 1$, where $\kappa$ is a representative wavenumber of the acoustic field, then the integrals in Eq. (29) are zero from the Reimann-Lebesque lemma, ${ }^{16}$ and the pressuredilatation is zero. (Note that similar arguments were made by Sarkar et al. ${ }^{13}$ in examining the long-time behavior of acoustics in isotropic turbulence.) In this case the pressure and velocity fields reach an equilibrium. However, the solutions differ somewhat from those in the previous section, because it is not assumed that the initial flow field is in acoustic equilibrium. Rather, acoustic equilibrium is achieved on an acoustic time scale, which in the limit considered is short compared to the mean-flow deformation time scale. With this approximation the integrals in Eqs. (28)-(32) involving the $\sin ()$ and $\cos ()$ terms drop out resulting in

$$
\begin{aligned}
& \overline{d^{\prime} d^{\prime}}(t)=\frac{\left[\overline{d^{\prime} d^{\prime}}(0)+\overline{\nabla p^{\prime} \cdot \nabla p^{\prime}}(0) /\left(\rho_{0} c_{0}\right)^{2}\right]}{2\left(1+S_{0} t\right)^{4}} \\
& =\frac{\overline{d^{\prime} d^{\prime}}(0)}{\left(1+S_{0} t\right)^{4}}\left[\frac{1}{2}+\frac{1}{2 F_{0}^{\prime}}\right], \\
& \overline{p^{\prime} d^{\prime}}(t)=0 \text {, } \\
& \overline{p^{\prime} p^{\prime}}(t)=\frac{\left[\overline{p^{\prime} p^{\prime}}(0)+\left(\rho_{0} c_{0}\right)^{2} \overline{u_{i}^{d^{\prime}} u_{i}^{d^{\prime}}}(0)\right]}{2\left(1+S_{0} t\right)^{10}} \\
& =\frac{\overline{p^{\prime} p^{\prime}}(0)}{\left(1+S_{0} t\right)^{10}}\left[\frac{1}{2}+\frac{F_{0}}{2}\right] \text {, } \\
& \overline{\boldsymbol{\nabla} p^{\prime} \cdot \boldsymbol{\nabla} p^{\prime}}(t)=\frac{\left[\overline{\nabla p^{\prime} \cdot \boldsymbol{\nabla} p^{\prime}}(0)+\left(\rho_{0} c_{0}\right)^{2} \overline{d^{\prime} d^{\prime}}(0)\right]}{2\left(1+S_{0} t\right)^{12}} \\
& =\frac{\overline{\boldsymbol{\nabla} p^{\prime} \cdot \nabla_{p^{\prime}}}(0)}{\left(1+S_{0} t\right)^{12}}\left[\frac{1}{2}+\frac{F_{0}^{\prime}}{2}\right], \\
& \overline{u_{i}^{d^{\prime}} u_{i}^{d^{\prime}}}(t)=\frac{\left[\overline{u_{i}^{d^{\prime}} u_{i}^{d^{\prime}}}(0)+\overline{p^{\prime} p^{\prime}}(0) /\left(\rho_{0} c_{0}\right)^{2}\right]}{2\left(1+S_{0} t\right)^{2}} \\
& =\overline{\frac{u_{i}^{d^{\prime}} u_{i}^{d^{\prime}}}{\left(1+S_{0} t\right)^{2}}}\left[\frac{1}{2}+\frac{1}{2 F_{0}}\right],
\end{aligned}
$$

where $F_{0}$ and $F_{0}^{\prime}$ are the initial values of the acoustic equilibrium parameters. Note that if the initial flow field is in acoustic equilibrium, these formulas reduce to those for the previous case. Even if the initial conditions are not in acoustic equilibrium, the above solutions give $F=1$ and $F^{\prime}=1$, so that the flow comes into equilibrium within a time scale that is short compared to the time scale of the mean deformation.

\section{E. Pressure released limit}

The other limiting case is where $S_{0} /\left(c_{0} \kappa\right) \gg 1$. This case corresponds to the "pressure-released" limit referred to by Jacquin et al. ${ }^{3}$ and Cambon et al. ${ }^{4}$ (The pressure-released RDT will be henceforth denoted by PR RDT.) In this case the $\cos ()$ and $\sin ()$ terms in the integrals in Eqs. (28)-(32) can be approximated for small values of the argument as one and zero respectively. An alternate approach is to begin with the RDT equations (12)-(13) and to neglect all the terms on the right hand side that are not proportional to the mean strain rate; this assumes that the time scale of the mean flow is small compared to the acoustic time scale. Doing so, one obtains (for general values of $\gamma$ )

$$
\begin{aligned}
& \overline{d^{\prime} d^{\prime}}(t)=\frac{\overline{d^{\prime} d^{\prime}}(0)}{\left(1+S_{0} t\right)^{4}}, \\
& \overline{p^{\prime} d^{\prime}}(t)=\frac{\overline{p^{\prime} d^{\prime}}(0)}{\left(1+S_{0} t\right)^{3 \gamma+2}}, \\
& \overline{p^{\prime} p^{\prime}}(t)=\frac{\overline{p^{\prime} p^{\prime}}(0)}{\left(1+S_{0} t\right)^{6 \gamma}}, \\
& \overline{\boldsymbol{\nabla} p^{\prime} \cdot \nabla p^{\prime}}(t)=\frac{\overline{\nabla p^{\prime} \cdot \nabla p^{\prime}}(0)}{\left(1+S_{0} t\right)^{6 \gamma+2}}, \\
& \overline{u_{i}^{d^{\prime}} u_{i}^{d^{\prime}}}(t)=\frac{\overline{u_{i}^{d^{\prime}} u_{i}^{d^{\prime}}}(0)}{\left(1+S_{0} t\right)^{2}} \text {. }
\end{aligned}
$$

While for a monatomic gas the solutions reduce to the same form as that for a field initially in acoustic equilibrium (except for the pressure dilatation, which here is nonzero), the flow field here does not have to be in acoustic equilibrium. The acoustic equilibrium parameters are given by

$$
F(t)=\frac{F_{0}}{\left(1+S_{0} t\right)^{5-3 \gamma}} \quad \text { and } F^{\prime}(t)=\frac{F_{0}^{\prime}}{\left(1+S_{0} t\right)^{5-3 \gamma}} .
$$

For a monatomic gas $(\gamma=5 / 3)$ these parameters do not change from their initial values, and the flow field remains in equilibrium or non-equilibrium depending on its initial condition. However, for a gas with $\gamma \neq 5 / 3$ the flow field is driven away from equilibrium even if it is initially in acoustic equilibrium. For a diatomic gas $(\gamma=7 / 5), F$ and $F^{\prime}$ become large for a compressive strain $\left(S_{0}<0\right)$, and the acoustic field becomes dominated by velocity fluctuations.

Another statistic of interest is the turbulent Mach number, $M_{T}$, defined by $M_{T}^{2}=\overline{u_{i}^{\prime} u_{i}^{\prime}} / c^{2}$, where $c^{2}=\gamma \bar{p} / \bar{\rho}$. The velocity variance is the sum of that due to the dilatational velocity, $\overline{u_{i}^{d^{\prime}} u_{i}^{d^{\prime}}}$ given in Eq. (51) and the solenoidal velocity, $u_{i}^{s^{\prime}} u_{i}^{s^{\prime}}$ given in Eq. (19). The turbulent Mach number is then given by

$$
M_{T}^{2}(t)=\frac{M_{T}^{2}(0)}{\left(1+S_{0} t\right)^{5-3 \gamma}} .
$$

One sees that for a monatomic gas the turbulent Mach number does not change. However, for a diatomic gas the turbulent Mach number increases during a compressive strain.

\section{COMPARISON WITH DNS}

The direct numerical simulations were done using the code of Blaisdell et al. ${ }^{8}$ The program uses a pseudo-spectral Fourier method for spatial derivatives and a third-order accurate, compact storage Runge-Kutta time advancement 
TABLE I. Initial parameters for the simulations.

\begin{tabular}{|c|c|c|c|c|c|c|c|c|c|c|c|}
\hline Case & $M_{T 0}$ & $S_{0}^{\star}$ & $\Delta m_{0}$ & $S_{\omega 0}^{\star}$ & $\Delta m_{0}^{*}$ & $R e_{T 0}$ & $\chi_{\varepsilon 0}$ & $\left(\rho_{\mathrm{rms}} / \bar{\rho}\right)_{0}$ & $\left(p_{\mathrm{rms}} / \bar{p}\right)_{0}$ & $F_{0}^{\prime}$ & $C_{p d 0}$ \\
\hline I1 & 0.10 & - & - & - & - & 200 & 0.013 & 0 & $1.2 \mathrm{e}-3$ & $1 \mathrm{e}+10$ & $5 e-7$ \\
\hline S1a & 0.099 & 200 & 19.9 & 14.3 & 1.4 & 198 & $7.5 e-3$ & $6.4 \mathrm{e}-3$ & 0.011 & 1.00 & -0.96 \\
\hline $\mathrm{S} 1 \mathrm{~b}$ & 0.097 & 200 & 19.4 & 14.7 & 1.4 & 187 & $7.4 \mathrm{e}-3$ & $8.0 \mathrm{e}-3$ & 0.013 & 0.82 & $6.5 e-3$ \\
\hline S1c1 & 0.091 & 200 & 18.2 & 16.2 & 1.5 & 153 & $6.5 e-3$ & $7.4 \mathrm{e}-3$ & 0.012 & 0.82 & $1.4 \mathrm{e}-3$ \\
\hline $\mathrm{S} 1 \mathrm{c} 2$ & 0.091 & 664 & 60.5 & 53.9 & 4.9 & 153 & $6.5 e-3$ & $7.4 \mathrm{e}-3$ & 0.012 & 0.82 & $1.4 \mathrm{e}-3$ \\
\hline $\mathrm{S} 1 \mathrm{c} 3$ & 0.091 & 2000 & 182.2 & 162.4 & 14.8 & 153 & $6.5 e-3$ & $7.4 \mathrm{e}-3$ & 0.012 & 0.82 & $1.4 \mathrm{e}-3$ \\
\hline $\mathrm{I} 2$ & 0.40 & - & - & - & - & 200 & 0.13 & 0 & $1.2 \mathrm{e}-3$ & $5 e+11$ & 0.0 \\
\hline $\mathrm{S} 2 \mathrm{a}$ & 0.38 & 200 & 76.7 & 15.2 & 5.8 & 188 & 0.078 & 0.081 & 0.13 & 0.93 & -0.95 \\
\hline $\mathrm{S} 2 \mathrm{~b} 1$ & 0.30 & 60 & 18.2 & 6.4 & 1.9 & 96 & 0.077 & 0.080 & 0.13 & 0.87 & 0.012 \\
\hline $\mathrm{S} 2 \mathrm{~b} 2$ & 0.30 & 200 & 60.5 & 21.3 & 6.4 & 96 & 0.077 & 0.080 & 0.13 & 0.87 & 0.012 \\
\hline S2b3 & 0.30 & 2000 & 605 & 212.5 & 63.8 & 96 & 0.077 & 0.080 & 0.13 & 0.87 & 0.012 \\
\hline $\mathrm{S} 3 \mathrm{a}$ & 0.01 & 20 & 0.2 & 1.4 & 0.014 & 200 & 0.013 & 0 & $1.1 \mathrm{e}-3$ & $1 e+10$ & $-6 e-7$ \\
\hline $\mathrm{S} 3 \mathrm{~b}$ & 0.01 & 200 & 2.0 & 14.2 & 0.142 & 200 & 0.013 & 0 & $1.1 \mathrm{e}-3$ & $1 e+10$ & $-4 e-7$ \\
\hline $\mathrm{S} 3 \mathrm{c}$ & 0.10 & 200 & 20 & 14.2 & 1.42 & 200 & 0.013 & 0 & $1.2 \mathrm{e}-3$ & $1 e+10$ & $5 e-7$ \\
\hline S3d & 0.10 & 2000 & 200 & 142.3 & 14.2 & 200 & 0.013 & 0 & $1.2 \mathrm{e}-3$ & $1 e+10$ & $5 e-7$ \\
\hline I4 & 0.40 & - & - & - & - & 2156 & 0.13 & 0.13 & 0.13 & 1.70 & -0.027 \\
\hline $\mathrm{S} 4 \mathrm{a}$ & 0.33 & 203 & 67 & 8.5 & 2.8 & 649 & 0.12 & 0.11 & 0.15 & 0.83 & 0.011 \\
\hline $\mathrm{S} 4 \mathrm{~b}$ & 0.33 & 2031 & 670 & 85.2 & 28.1 & 649 & 0.12 & 0.011 & 0.15 & 0.83 & 0.011 \\
\hline
\end{tabular}

scheme. Unlike the incompressible flow code of Rogallo, ${ }^{10}$ the current method is not exact in the limit of rapid distortions for arbitrarily large time steps. Therefore, the time step for each simulation was reduced until a time-accurate solution was obtained. Each of the simulations was performed on a $128 \times 128 \times 128$ grid.

Three uncompressed decaying isotropic turbulence simulations, I1, I2, and I4, were performed in order to generate initial conditions for three series of compression runs, S1, S2 and S4 respectively (see Table I). In this way the initial flow fields for the strained cases are developed to some extent. Compression run series S3 starts from random initial conditions and, therefore, does not have a corresponding isotropic decay case. The initial conditions for the simulations were generated from random numbers with a specified power spectrum. The form of the spectrum for fluctuating quantities is taken to be $E(k)$ $=A k^{4} \exp \left[-2\left(k / k_{p}\right)^{2}\right]$, where $k_{p}$ is the wavenumber at which the peak in the spectrum occurs and $A$ is a constant that is adjusted to obtain the desired variance (square of the turbulent Mach number for the case of velocity fluctuations).

Simulations I1, I2 and series S3 have $k_{p}=12$. They also use $\gamma=5 / 3$ and begin far from acoustic equilibrium by having some dilatational velocity fluctuations but no density or pressure fluctuations. The other parameters that determine the initial conditions of each simulation are shown in Table I. The nondimensional strain rate is $S^{\star}=\bar{\rho}|S| q^{2} / \varepsilon$, where $S$ is the mean strain rate, $q^{2}=\overline{u_{i}^{\prime} u_{i}^{\prime}}$, and $\varepsilon$ is the dissipation rate of turbulent kinetic energy per unit volume. This parameter gives the ratio of the time scale of the large energy containing turbulent eddies to that of the mean flow. The subscript 0 denotes the initial value. The parameter $\Delta m=S^{\star} M_{T}$ $=|S|(\ell / c)$, where $\ell$ is a turbulent length scale, was introduced by Durbin and Zeman ${ }^{2}$ in defining the HF limit. It is the ratio of the time for an acoustic wave to travel the distance of a turbulent length scale to the mean flow deformation time. In terms of a representative wavenumber $\Delta m$ $\propto S /(c \kappa)$, which was used in section II to distinguish be- tween the HF and PR limits. Thus for the HF limit $\Delta m \ll 1$, and for the PR limit $\Delta m \gg 1$. A measure of the mean flow time scale to that of the small, dissipation eddies is given by

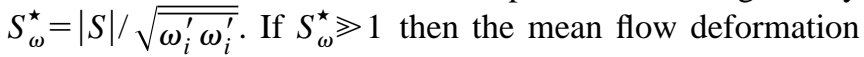
is rapid compared to the dissipation processes. If $S_{\omega}^{\star}=\mathscr{Q}(1)$, the mean flow deformation may be rapid compared to the large length scales but not rapid compared to the small length scale eddies. Another time scale parameter involving the small scales is $\Delta m^{*}=S_{\omega}^{\star} M_{T}$, which was introduced by Cambon et al. ${ }^{4}$ It is shown below that although several simulations have $\Delta m^{*}=\mathscr{Q}(1)$, they are still rapid with respect to the small scales. The turbulent Reynolds number is $\operatorname{Re}_{T}=\bar{\rho}^{2} q^{4} / \mu \varepsilon$. The parameter $\chi_{\varepsilon}=\varepsilon_{d} /\left(\varepsilon_{s}+\varepsilon_{d}\right)$ is the ratio of the dilatational dissipation to the total dissipation. It gives a measure of the strength of the dilatation relative to the vorticity. The scaled r.m.s. density and pressurefluctuation levels are $\rho_{\mathrm{rms}} / \bar{\rho}=\left(\overline{\rho^{\prime} \rho^{\prime}}\right)^{1 / 2} / \bar{\rho}$ and $p_{\text {rms }} / \bar{p}=\left(\overline{p^{\prime} p^{\prime}}\right)^{1 / 2} / \bar{p}$. A measure of the equilibrium state of the initial acoustic field is given by $F^{\prime}$ and $C_{p d}=\overline{p^{\prime} d^{\prime}} /\left[\left(\overline{p^{\prime} p^{\prime}}\right)\left(\overline{d^{\prime} d^{\prime}}\right)\right]^{1 / 2}$, which is the pressuredilatation correlation coefficient.

Case I4 differs from the other initial-condition runs in that $\gamma=7 / 5$ and the flow starts close to acoustic equilibrium. Also, $k_{p}=4$ and $\mathrm{Re}_{T}$ is higher than for the other isotropic turbulence simulations; however, this is not expected to be significant to the behavior of the acoustic field discussed below.

The RDT solution for the vorticity mode discussed in section III A yielded formulas for the evolution of the enstrophy and the solenoidal velocity variance. The DNS code does not compute the solenoidal velocity due to the computational expense involved; however, since the dilatational velocity is relatively weak compared to the solenoidal velocity, and because the dilatational velocity scales in the same way as the solenoidal velocity for the cases of acoustic equilibrium and PR RDT, the evolution of the turbulent kinetic energy, $k$, can be considered instead. Accounting for the 


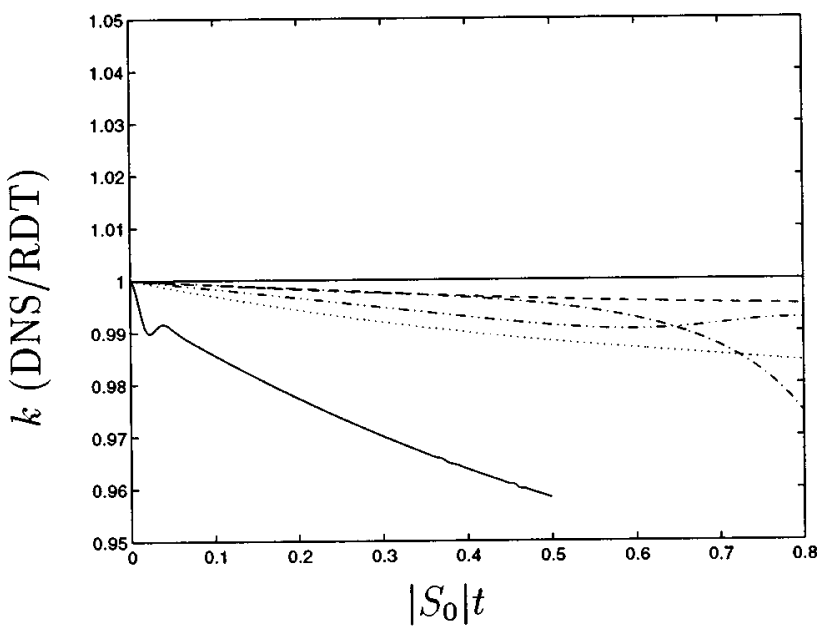

FIG. 2. Ratio of the turbulent kinetic energy from the DNS to the approximate RDT result (54) for cases S3a with $S_{0}^{\star}=20, \ldots$; S2b1 with $S_{0}^{\star}=60.3$, …...; S4a with $S_{0}^{\star}=203,-\cdot-$; S1a with $S_{0}^{\star}=200,-\cdots-$; S1c1 with $S_{0}^{\star}=200,--\cdot$

variation of the mean density and neglecting the dilatational velocity or assuming acoustic equilibrium or PR RDT, one then obtains the approximate RDT result

$$
k(t)=\frac{k(0)}{\left(1+S_{0} t\right)^{5}} .
$$

A comparison of the turbulent kinetic energy between the DNS results and this RDT prediction is given in Fig. 2. Results are shown for a number of simulations that have relatively low values of $S_{0}^{\star}$ and $S_{\omega 0}^{\star}$ and, therefore, would be least likely to show good agreement. Simulation S3a, with $S_{0}^{\star}=20$, which is significantly lower than that of the other simulations, has the largest error, as expected. This simulation was stopped at $\left|S_{0}\right| t=0.5$ rather than 0.8 because of the large number of time steps needed. The other simulations display differences with the RDT result that are on the order of $2 \%$ or less, indicating that the mean flow strain is rapid

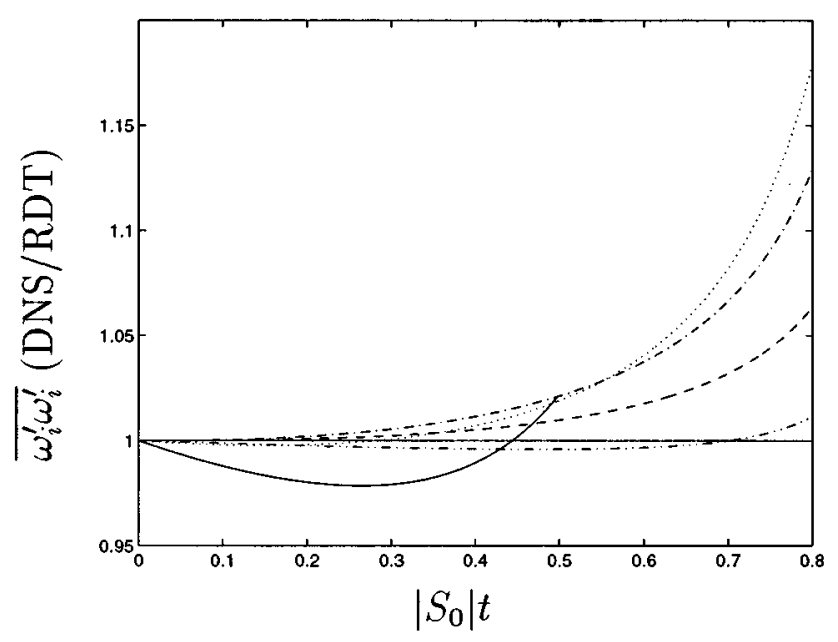

FIG. 3. Ratio of the enstrophy from the DNS to the RDT result (17) for cases S3a with $S_{\omega 0}^{\star}=1.4, \ldots ;$ S2b1 with $S_{\omega 0}^{\star}=6.4, \cdots \ldots \ldots$; S4a with $S_{\omega 0}^{\star}=8.5,-\cdot-;$ S1a with $S_{\omega 0}^{\star}=14.3,-\cdot-;$; S1c1 with $S_{\omega 0}^{\star}=16.2,--\cdot$
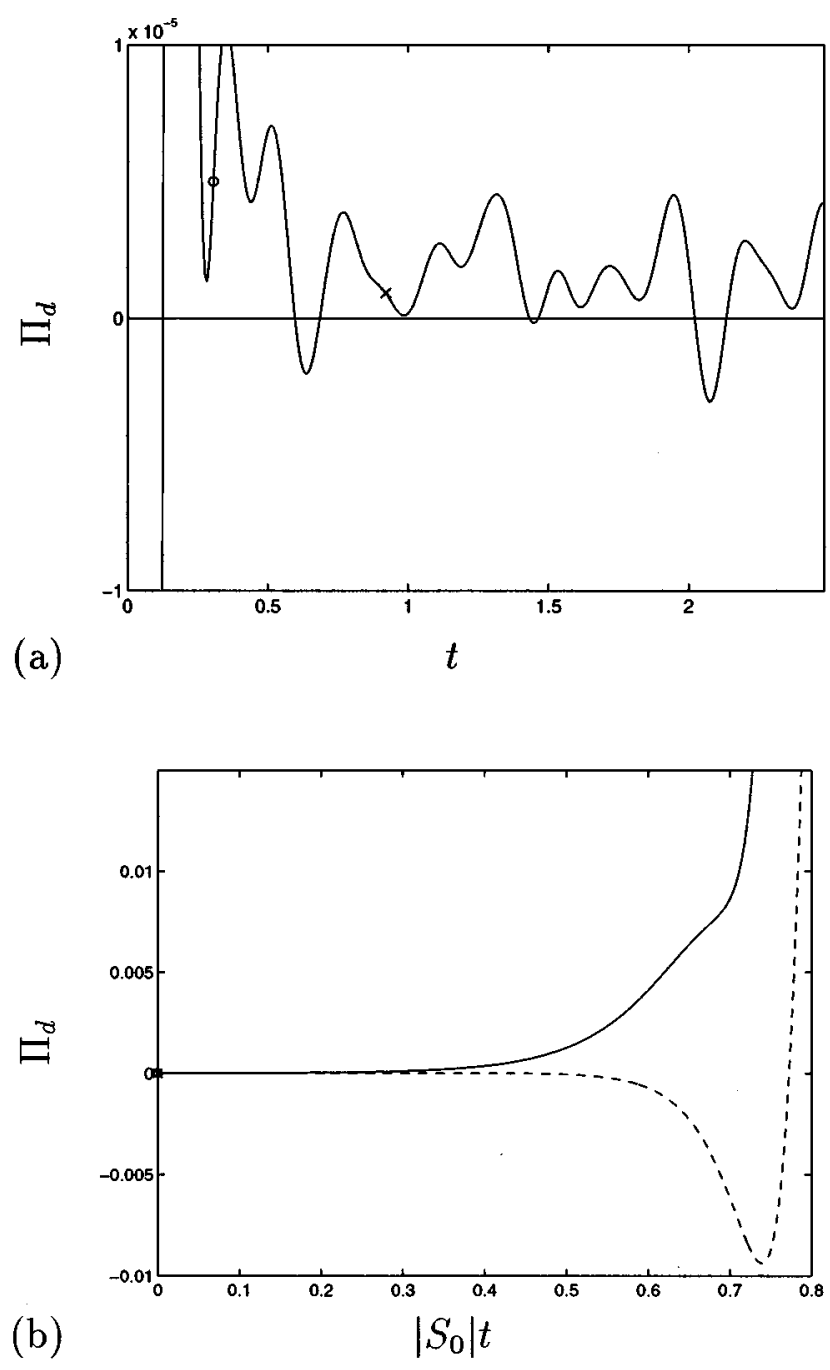

FIG. 4. Pressure-dilatation for (a) the isotropic decay run I1, and (b) strained cases S1b, - , and S1c1, ---. (The initial conditions for S1b and S1c1 are marked by $\bigcirc$ and $\times$ respectively.)

relative to the time scales of the large, energy containing eddies. For most of the time simulated the results scale with $S_{0}^{\star}$, in that the more rapid cases show better agreement between the DNS and RDT results. There is some variation at later time. This is believed to be due to differences in initial conditions and the effect of the acoustic mode on the turbulent kinetic energy.

In Fig. 3 the enstrophy for these same simulations is compared to the RDT prediction given in equation (17). Again, simulation S3a has the most noticeable difference. Since this simulation has a $S_{\omega 0}^{\star}$ of order one, the mean flow strain is not rapid compared to the time scale of the smallscale eddies. The other simulations show good agreement between the DNS and RDT result up to $\left|S_{0}\right| t=0.5$. Beyond that the discrepancies grow, becoming at $\left|S_{0}\right| t=0.8$ on the order of $15-20 \%$. The error seen in the enstrophy is greater than that found in the turbulent kinetic energy, as is expected because of the relative difference in the time scales of the small and large eddies. The magnitude of the error in the RDT prediction of the enstrophy is generally consistent with the value of $S_{\omega 0}^{\star}$ in the simulations; this is true at early times 

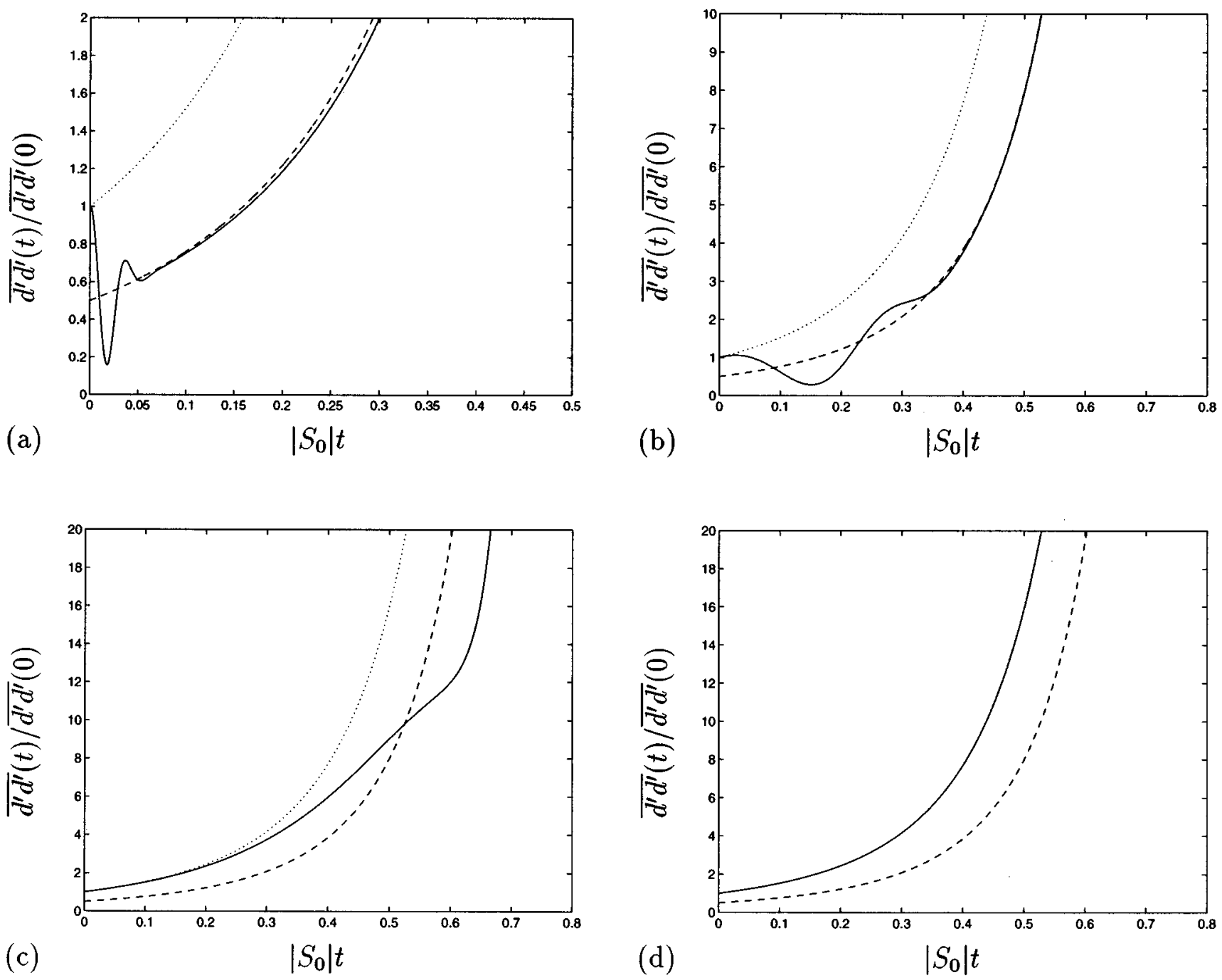

FIG. 5. Evolution of the scaled mean squared fluctuating dilatation, $\overline{d^{\prime} d^{\prime}}(t) / \overline{d^{\prime} d^{\prime}}(0)$, from the DNS, - , compared to the high frequency RDT solution, ---and the pressure-released RDT solution, $\cdots \cdots$..., for (a) case S3a with $\Delta m_{0}=0.2$, (b) case S3b with $\Delta m_{0}=2$, (c) case S3c with $\Delta m_{0}=20$, and (d) case S3d with $\Delta m_{0}=200$.

also, although it is difficult to discern this from the figure shown. An exception is case S1a, which has a much higher discrepancy at early times and a much lower one later, relative to the other large- $S_{\omega 0}^{\star}$ simulations considered. This difference is believed to be due to variations in initial conditions and nonlinear interactions between the vortical and acoustic modes.

One point that comes from the analysis of the acoustic mode is that the pressure-dilatation is highly dependent on initial conditions. To demonstrate this point two runs were made with different initial states of the acoustic field. Figure 4(a) shows the pressure-dilatation for the unstrained simulation case I1. There is a strong initial transient followed by smaller oscillations. The pressure-dilatation is more positive than negative during the decay, which has been observed and explained in previous studies of isotropic turbulence. ${ }^{8,12-14,17}$ The two symbols in Fig. 4(a) mark the initial conditions for the strained-flow simulations S1b and S1c1. Note that for simulation S1b the pressure-dilatation is positive and increasing while for simulation $\mathrm{S} 1 \mathrm{c} 1$ it is positive and decreas- ing. This difference in the initial state of the acoustic field leads to very different behavior in the evolution of the pressure-dilatation correlation as shown in Fig. 4(b). The fact that $\Pi_{d}$ is strongly dependent on initial conditions means that algebraic turbulence models for pressure-dilatation, which are not sensitive to initial conditions, should not be expected to give accurate results in general for such flows (see below).

Two limiting cases of the exact solution are given in section II, the high frequency (HF) and the pressure-released (PR) limits. The solutions for these two cases differ only if the initial conditions are out of equilibrium. In order to contrast these two limits a series of simulations was done in which the initial conditions were far from equilibrium and the ratio of the mean flow to acoustic time scales was varied.

In series S3a-d the parameter $\Delta m_{0}$ was varied from 0.2 to 200. The simulations were made without allowing the initial isotropic turbulence, which were taken from randomly generated fields, to decay. The initial density fluctuations were zero and the pressure fluctuations were small, so the 

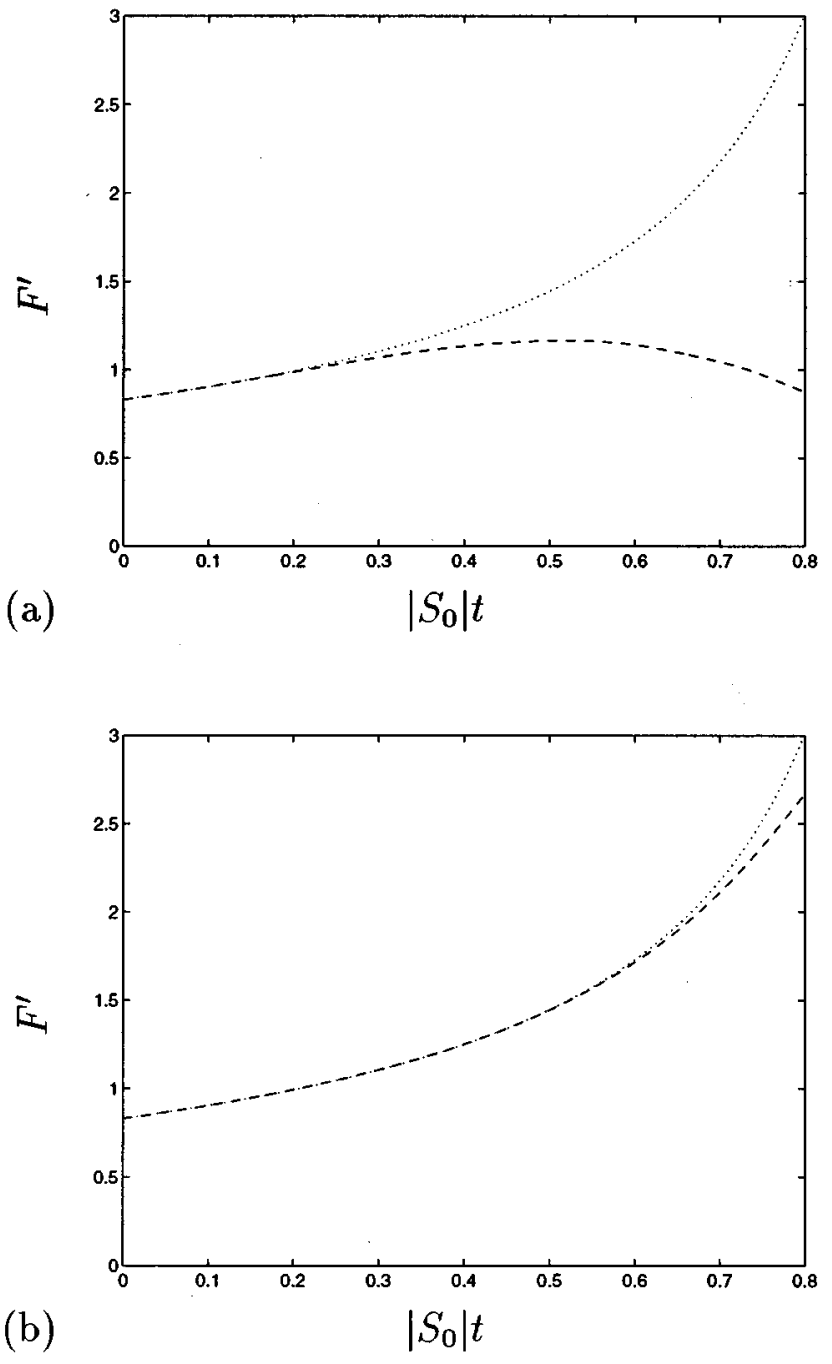

FIG. 6. Evolution of the acoustic equilibrium parameter $F^{\prime}$ for a diatomic gas $(\gamma=7 / 5)$, comparing DNS, ---, and pressure-released RDT, $\cdots \cdots \cdots$, for (a) case S4a with $\Delta m_{0}=67$, and (b) case S4b with $\Delta m_{0}=670$.

initial values of $F^{\prime}$ were very large. Equations (42) and (47) indicate a difference of a factor of 2 for the dilatation in the HF and PR limits. The dilatation for case S3a, for which $\Delta m_{0}=0.2$, is shown in Fig. 5(a). There are some oscillations in the evolution, but the turbulence moves towards equilibrium very quickly and for later times the HF solution matches the DNS data very well. Case S3b has $\Delta m_{0}=2$ and, as shown in Fig. 5(b), it takes a longer nondimensional time for the acoustics to come into equilibrium, at which time the DNS and HF RDT results agree. For case S3c, $\Delta m_{0}=20$ and it appears that the acoustic time scale is on the same order as the deformation time scale, so that the acoustic field oscillates but does not come to equilibrium within the time of the simulation, as shown in Fig. 5(c). Case S3d has $\Delta m_{0}=200$. Here the time scale of the mean flow deformation is much shorter than that of the acoustics and the acoustic field does not have a chance to move toward equilibrium. As shown in Fig. 5(d), the PR solution matches the DNS data almost exactly for the time considered, so that the curves are indistinguishable.
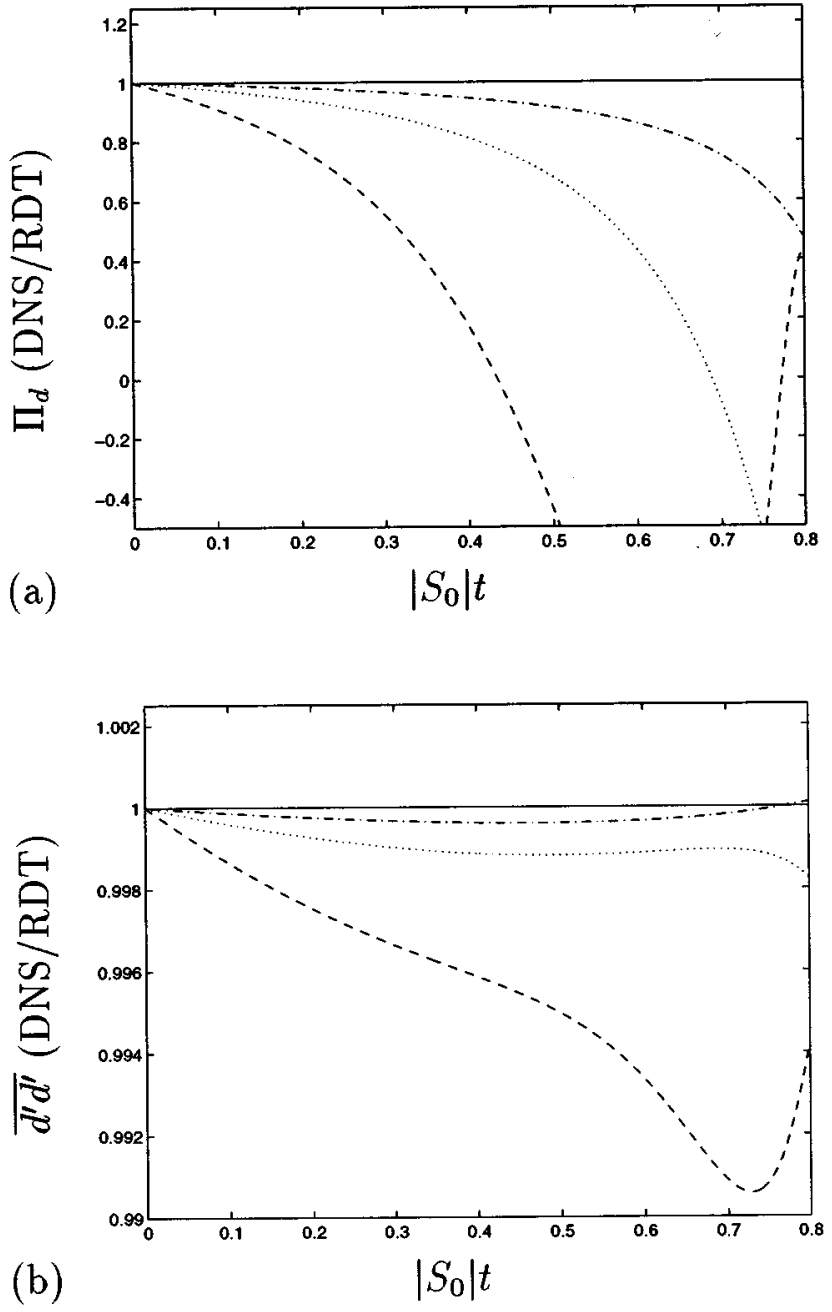

FIG. 7. Ratio of DNS to pressure-released RDT results for (a) the pressuredilatation, $\Pi_{d}$, and (b) the mean squared fluctuating dilatation, $\overline{d^{\prime} d^{\prime}}$, for cases S1c1 with $\Delta m_{0}=18.2,---; \mathrm{S} 1 \mathrm{c} 2$ with $\Delta m_{0}=60.5, \cdots \ldots \ldots$; and S1c3 with $\Delta m_{0}=182.2,-\cdot-$.

The results presented in Fig. 5 show that the acoustic field tends toward equilibrium provided the deformation is sufficiently slow. However, as noted in section II, the PR solution indicates that for non-monatomic gases the acoustic field is driven away from equilibrium. For simulation S4a $\gamma=7 / 5$ and $\Delta m_{0}=67$. The evolution of $F^{\prime}$ is shown in Fig. 6(a). The acoustic field is initially close to equilibrium, with $F_{0}^{\prime}=0.83$ and $C_{p d}$ small, and as the flow develops $F^{\prime}$ increases following the PR predictions. However, at later times the DNS results diverge from the PR solution and the acoustic field tends back toward equilibrium. In order to maintain agreement with the PR solution it is necessary to increase $\Delta m_{0}$. This is shown in Fig. 6(b) for simulation S4b, for which $\Delta m_{0}=670$.

As illustrated in Figs. 6(a) and 6(b), better agreement between the DNS results and the PR limit is found as $\Delta m_{0}$ is increased. This is also seen for the pressure-dilatation and the dilatation, as shown in Figs. 7(a) and 7(b) for cases S1c1-3. For $\Delta m_{0}=18$ the PR result for the pressure-dilatation is significantly in error after a short time. As $\Delta m_{0}$ is increased to 182 the PR result is accurate for longer $\left|S_{0}\right| t$. The dilatation, shown in Fig. 7(b), displays a similar trend; however, the 

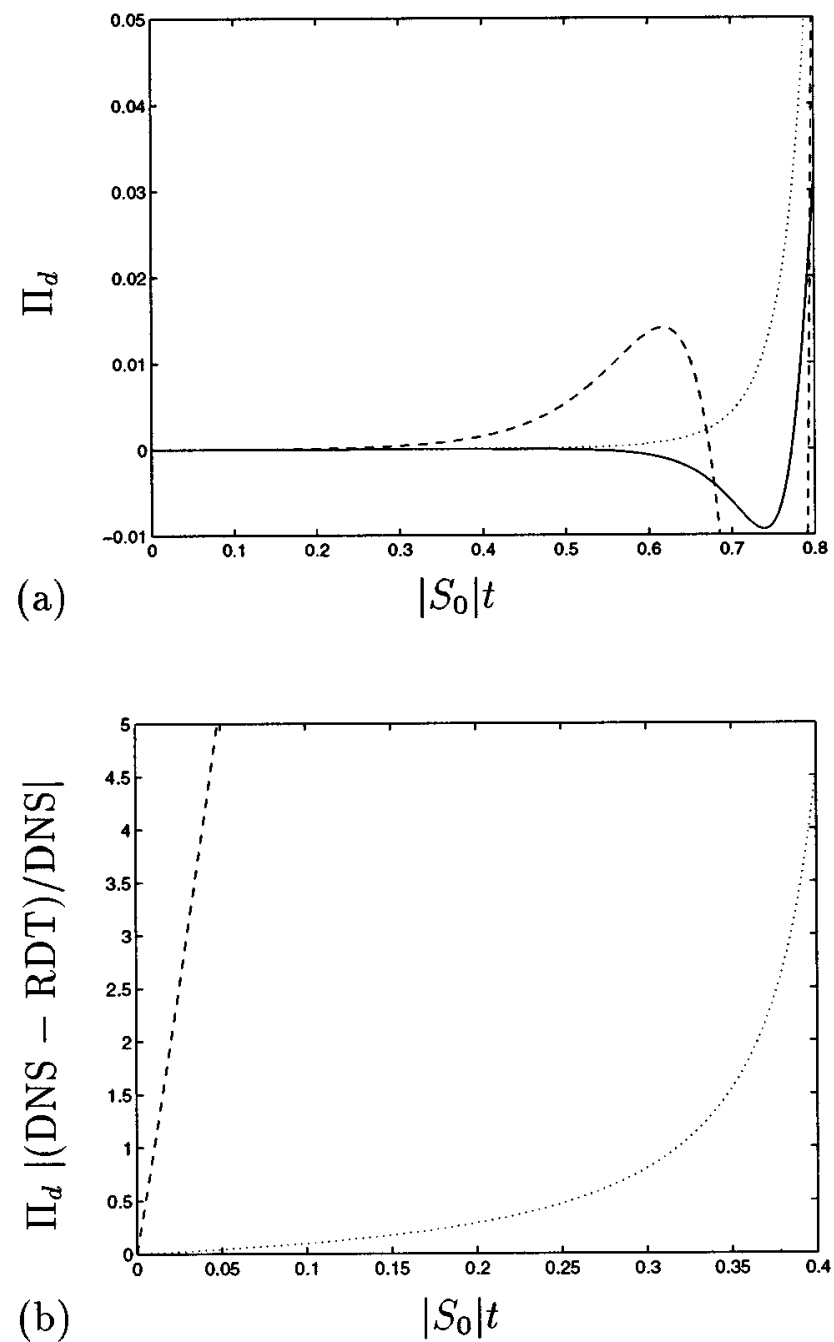

FIG. 8. (a) Pressure-dilatation for case S1c1 (with $\Delta m_{0}=18.2$ and the acoustic field near equilibrium) from the DNS, - , the exact RDT solution, ---, and pressure-released RDT, ........ (b) Relative error in the pressuredilatation for the exact RDT solution, ---, and pressure-released RDT, ........

magnitude of the error is very small-less than $1 \%$ for all three cases. For cases S2b1-3 (results not shown), in which the initial turbulent Mach number is higher, the same trends are observed, but the magnitude of the error in the pressuredilatation is lower and error in the dilatation is greater.

The HF and PR RDT solutions are valid in the limit of $\Delta m_{0}$ being small and large respectively. For intermediate values of $\Delta m_{0}$, neither limiting case holds; however, the exact solution presented in section II is expected to be correct over the full range of $\Delta m_{0}$. In order to use this solution the initial spectra are needed for the pressure variance, the pressure-dilatation, and the dilatation. These initial spectra were taken from the DNS flow fields and used to compute the integrals in Eqs. (28)-(32). The results for the pressuredilatation for case S1c1 (with $\Delta m_{0}=18$ ) are shown in Fig. 8(a) along with the DNS and PR RDT results. The PR RDT result cannot change sign and so a large error develops at later times. The exact solution can change sign as it accounts for the transfer of energy between the pressure and velocity fields; however, it is seen to be grossly in error. For the
TABLE II. Terms in the pressure-dilatation equation for the initial conditions of case $\mathrm{S} 1 \mathrm{c} 1$.

\begin{tabular}{lc}
\hline \hline Term & Value \\
\hline 1 & $-0.317744 \mathrm{e}-2$ \\
$1 \mathrm{a}$ & $-0.317666 \mathrm{e}-2$ \\
$1 \mathrm{~b}$ & $-0.784663 \mathrm{e}-6$ \\
2 & $-0.652143 \mathrm{e}-2$ \\
$2 \mathrm{a}$ & $-0.476499 \mathrm{e}-2$ \\
$2 \mathrm{~b}$ & $-0.175644 \mathrm{e}-2$ \\
3 & $0.968496 \mathrm{e}-2$ \\
$3 \mathrm{a}$ & $0.968523 \mathrm{e}-2$ \\
$3 \mathrm{~b}$ & $-0.266120 \mathrm{e}-6$ \\
4 & $1.34567 \mathrm{e}-3$ \\
$1+2+3$ & -0.00001391 \\
$1 \mathrm{a}+2 \mathrm{a}+3 \mathrm{a}$ & 0.00174358 \\
$1 \mathrm{~b}+2 \mathrm{~b}+3 \mathrm{~b}$ & -0.00175749 \\
\hline \hline
\end{tabular}

dilatation (not shown) the exact RDT solution has a small error relative to the DNS results, but the PR RDT solution is even closer to the DNS results.

The above results imply that the exact RDT solution is less accurate than the approximate PR RDT solution. Insight into this unexpected behavior is found by considering the pressure-dilatation in more detail. Figure 8(b) shows the relative error in the exact and PR RDT solutions for the pressure-dilatation at early times. One can see that the exact RDT begins with the wrong slope (non-zero slope in the relative error), so that the time derivative of the pressuredilatation is not faithfully captured.

To examine this point further, the transport equation for the pressure-dilatation was considered. This equation is derived in Ref. 17. The linearization used in the RDT analysis is equivalent to keeping terms that are second order in fluctuating quantities while neglecting higher-order terms. Isolating the second-order terms, neglecting the viscous and heat conduction terms, and assuming homogeneous turbulence subjected to isotropic strain, the equation for the pressuredilatation can be written as

$$
\begin{aligned}
& \frac{\bar{D}}{\bar{D} t}\left(\overline{p^{\prime} d^{\prime}}\right)=-(\gamma-1)\left[\bar{p}\left(\overline{\frac{\partial u_{i}^{\prime}}{\partial x_{i}} \frac{\partial u_{j}^{\prime}}{\partial x_{j}}}\right)+\overline{p^{\prime} \frac{\partial u_{i}^{\prime}}{\partial x_{i}} \frac{\partial u_{j}^{\prime}}{\partial x_{j}}}\right] \\
& \text { Term 1a,b } \\
& \left.-\left[\overline{p\left(\overline{\frac{\partial u_{i}^{\prime}}{\partial x_{j}} \frac{\partial u_{j}^{\prime}}{\partial x_{i}}}\right.}\right)+\overline{p^{\prime} \frac{\partial u_{i}^{\prime}}{\partial x_{j}} \frac{\partial u_{j}^{\prime}}{\partial x_{i}}}\right] \\
& \text { Term 2a,b } \\
& -\left[\frac{1}{\bar{\rho}}\left(\overline{\frac{\partial p^{\prime}}{\partial x_{i}} \frac{\partial p^{\prime}}{\partial x_{i}}}\right)+\left(\overline{\left.\frac{1}{\rho}-\frac{1}{\bar{\rho}}\right) \frac{\partial p^{\prime}}{\partial x_{i}} \frac{\partial p^{\prime}}{\partial x_{i}}}\right]\right. \\
& \text { Term 3a,b } \\
& -(3 \gamma+2)\left(\overline{p^{\prime} d^{\prime}}\right) S(t) \text {. } \\
& \text { Term } 4
\end{aligned}
$$


The terms in this equation were computed for the initial flow field used for simulation S1c1. The results are tabulated in Table II. It is found that the linearization is an excellent approximation for terms 1 and 3, but only a fair approximation for term 2, in which a $27 \%$ error is made. (Compare parts $a$ and $b$ for each.) This difference, however, does not explain the large discrepancy found in the comparison of the pressure-dilatation. If the first three terms are added together it is found that they nearly cancel. However, the sum of the first three linearized terms is a factor of 125 larger. Therefore, there is a delicate balance among the first three terms and the poor approximation of term 2 causes the right hand side of the pressure-dilatation equation to have a significant error.

In term $2 b$ the factor multiplying the fluctuating pressure is recognized as the right hand side of the Poisson equation for the slow pressure in an incompressible flow. In the present analysis, term $2 \mathrm{~b}$ is neglected because it involves higher order fluctuations. However, perhaps a more accurate analysis could be made that accounts for such terms in the limit of nearly incompressible flow. ${ }^{18}$

The exact RDT solution is found to be more accurate if the linear terms are dominant. Simulation S1a uses initial conditions taken from the early transient of the isotropic decay run I1, at the point when the pressure-dilatation reaches its most negative value. Although $F_{0}^{\prime}=1.0, C_{p d 0}$ is large (close to 1) and so the acoustic field is out of equilibrium and $F^{\prime}$ quickly changes. The results for the pressure-dilatation are shown in Fig. 9(a). Here the exact RDT solution more closely follows the behavior found in the DNS. The relative error is given in Fig. 9(b), and the exact solution is now more accurate than the PR RDT solution. Therefore, the exact RDT solution is seen to be correct for intermediate values of $\Delta m$; however, it is limited to situations in which the acoustic fluctuations are far from equilibrium.

\section{CONCLUSION}

We have examined the RDT problem for compressible homogeneous turbulence under isotropic mean strain. An exact solution has been found. Simplified forms of the solution are given for two limiting cases - the high-frequency limit (acoustic time scale small compared to the mean deformation time scale) and the pressure-released or high strain rate limit (mean deformation time scale small compared to the acoustic time scale). The RDT solutions are compared to results from direct numerical simulations in order to demonstrate the range of validity of the RDT assumptions.

The RDT analysis shows that the vorticity and acoustic modes are decoupled. Although by definition all results of RDT - since they are the product of a linear analysis - are initial-condition dependent, the presence or absence of linear coupling between the various modes determines how quickly the turbulence "forgets" its initial state (Blaisdell et al. ${ }^{8}$ ). In this sense, RDT indicates the sensitivity to initial conditions of the general non-linear case. Because there is no linear coupling between the vortical and acoustic fields during a rapid isotropic strain, quantities of interest in turbulence modeling, such as the pressure-dilatation correlation, are
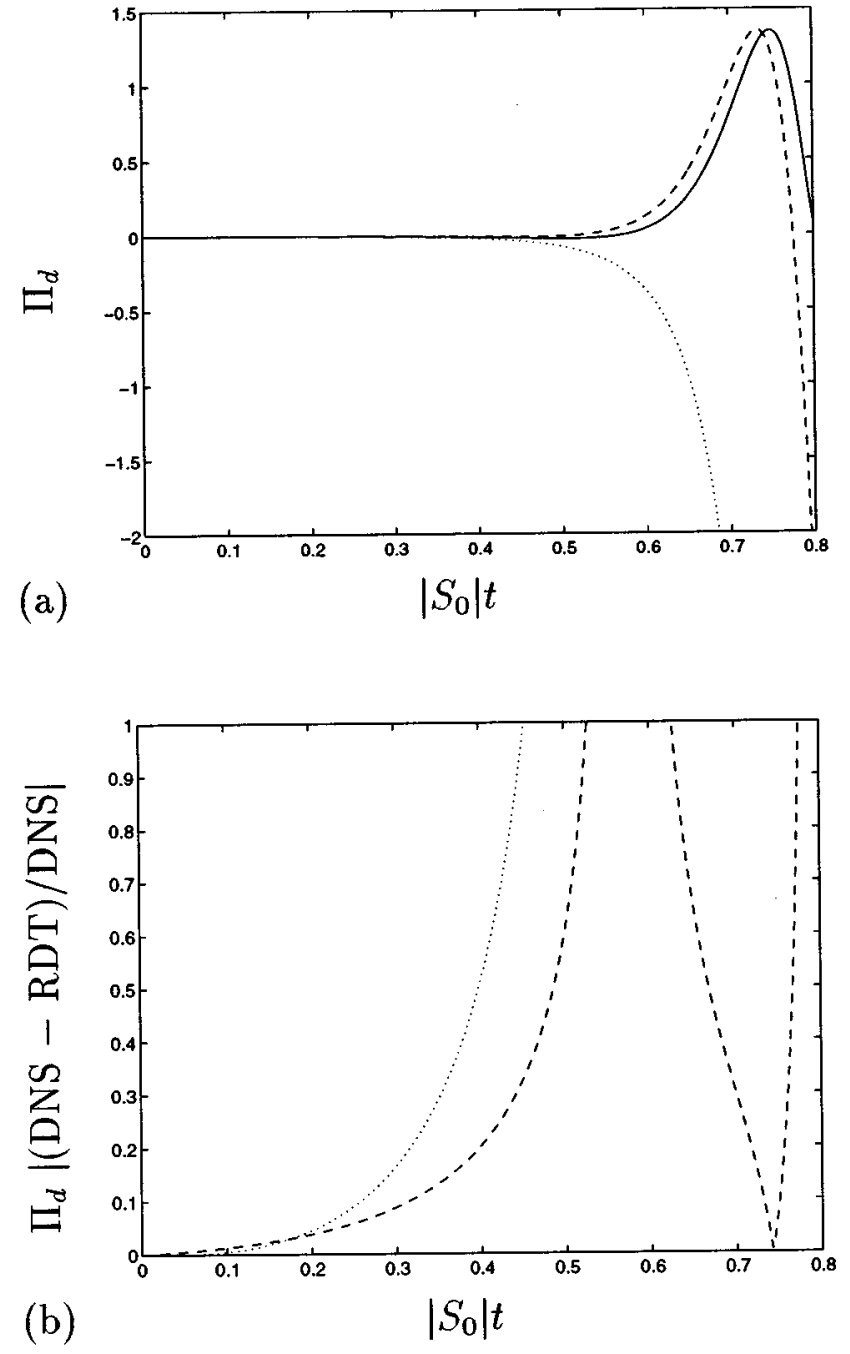

FIG. 9. (a) Pressure-dilatation for case S1a (with $\Delta m_{0}=19.9$ and the acoustic field far from equilibrium) from the DNS, - , the exact RDT solution, --- , and pressure-released RDT, $\cdots \cdots \cdots$. (b) Relative error in the pressuredilatation for the exact RDT solution, ---, and pressure-released RDT, n......

highly dependent on initial conditions. Therefore, algebraic turbulence models for these quantities, which are not sensitive to initial conditions, will not in general be accurate for this type of flow. In addition it is found that the initial acoustic equilibrium state significantly affects the development of the turbulence. For acoustic fields in equilibrium, the highfrequency and pressure-released limits give the same prediction for the statistics considered. However, for acoustic fields initially out of equilibrium the two limits differ. DNS results show that turbulence initially far from acoustic equilibrium will tend toward equilibrium; however, if the mean strain rate is large enough, the acoustic field will not have sufficient time to equilibrate.

In the pressure-released limit, the tendency toward acoustic equilibrium is dependent on the ratio of specific heats of the gas. For a monatomic gas the acoustic equilibrium state will not change from its initial condition, while for a diatomic gas the acoustic field will drift away from equilibrium and become dominated by velocity fluctuations. The 
direct numerical simulations show that these predictions are valid for short times, but later the acoustic field tends toward equilibrium. For larger strain rates, the above predictions are valid for longer nondimensional times.

For intermediate strain rates the acoustic field displays oscillations, which are not predicted by either of the limiting cases. The exact RDT solution is expected to be valid in this regime. However, comparisons with DNS indicate that a significant error is made due to nonlinear effects when the initial conditions are close to acoustic equilibrium. It is found that when the initial conditions are far from equilibrium, the exact RDT solution predicts the behavior accurately. Therefore, for intermediate strain rates RDT is only valid for initial conditions that are far from acoustic equilibrium, where linear effects dominate.

Because of the above restriction on the validity of RDT for intermediate strain rates, the RDT solution may be of limited utility as a means to directly evaluate turbulence models. However, this study should prove useful as an indication of the range of applicability of the RDT assumptions for turbulence modelers when they use RDT in the development of models for compressible flows.

\section{ACKNOWLEDGMENTS}

Computer time for this work was provided in part by the Numerical Aerodynamic Simulation program (NAS), the National Center for Super-computing Applications (NCSA), the Center for Advanced Computing Research (CACR), and the Purdue University Computing Center. Discussions with Dr. Claude Cambon were very helpful.

\section{APPENDIX: ACOUSTIC MODE SOLUTION FOR GENERAL $\gamma$}

An analytic solution for the acoustic mode for general values of $\gamma$ is possible, although it is more complicated than that for a monatomic gas. In this Appendix the solution for the Fourier coefficients for dilatation and pressure are found for general values of $\gamma$.

In Eq. (20) let $\tau=1 /\left(1+S_{0} t\right)$, which results in

$$
\tau^{2} \frac{d^{2} \breve{d}}{d \tau^{2}}+(5-3 \gamma) \tau \frac{d \breve{d}}{d \tau}+\left(\frac{c_{0} \kappa}{S_{0}}\right)^{2} \tau^{3(\gamma-1)} \breve{d}=0 .
$$

This equation can be solved in terms of Bessel functions (Ref. 19, formula 9.1.53), giving

$$
\begin{aligned}
\breve{d}(\tau)= & A \tau^{((3 / 2) \gamma-2)} J_{\nu}\left(\lambda \tau^{(3 / 2)(\gamma-1)}\right) \\
& +B \tau^{((3 / 2) \gamma-2)} Y_{\nu}\left(\lambda \tau^{(3 / 2)(\gamma-1)}\right),
\end{aligned}
$$

where $\nu=\left(\gamma-\frac{4}{3}\right) /(\gamma-1)$ and $\lambda=2 c_{0} \kappa /\left(3(\gamma-1)\left|S_{0}\right|\right)$.

Reverting back to the Fourier coefficient of the dilatation gives

$$
\begin{aligned}
\hat{d}(\kappa, t)= & A \frac{1}{\left(1+S_{0} t\right)^{(3 / 2) \gamma}} J_{\nu}\left(\frac{\lambda}{\left(1+S_{0} t\right)^{(3 / 2)(\gamma-1)}}\right) \\
& +B \frac{1}{\left(1+S_{0} t\right)^{(3 / 2) \gamma}} Y_{\nu}\left(\frac{\lambda}{\left(1+S_{0} t\right)^{(3 / 2)(\gamma-1)}}\right) .
\end{aligned}
$$

The Fourier coefficient of the pressure is found from Eq. (13). Making use of recurrence relations for the derivative of a Bessel function results in

$$
\begin{aligned}
\hat{p}(\kappa, t)= & -A \frac{\rho_{0} S_{0}}{\kappa^{2}}\left(\frac{c_{0} \kappa}{\left|S_{0}\right|}\right) \frac{1}{\left(1+S_{0} t\right)^{3 \gamma+(1 / 2)}} \\
& \times J_{\nu^{\prime}}\left(\frac{\lambda}{\left(1+S_{0} t\right)^{(3 / 2)(\gamma-1)}}\right)-B \frac{\rho_{0} S_{0}}{\kappa^{2}}\left(\frac{c_{0} \kappa}{\left|S_{0}\right|}\right) \\
& \times \frac{1}{\left(1+S_{0} t\right)^{3 \gamma+(1 / 2)}} Y_{\nu^{\prime}}\left(\frac{\lambda}{\left(1+S_{0} t\right)^{(3 / 2)(\gamma-1)}}\right),
\end{aligned}
$$

where $\nu^{\prime}=\nu-1=-1 /(3(\gamma-1))$.

The constants $A$ and $B$ can be put in terms of the initial values of the Fourier coefficients by setting $t=0$ in Eqs. (A3)-(A4) and solving. The resulting solution can be simplified (by making use of the Wronskian, ${ }^{19} W\left\{J_{\nu^{\prime}}, Y_{\nu^{\prime}}\right\}$ ), yielding

$$
\begin{aligned}
\hat{d}(\kappa, t)= & \frac{\pi}{3(\gamma-1)}\left(\frac{c_{0} \kappa}{\left|S_{0}\right|}\right)\left[Y_{\nu^{\prime}}(\lambda) J_{\nu}\left(\frac{\lambda}{\left(1+S_{0} t\right)^{(3 / 2)(\gamma-1)}}\right)\right. \\
& \left.-J_{\nu^{\prime}}(\lambda) Y_{\nu}\left(\frac{\lambda}{\left(1+S_{0} t\right)^{(3 / 2)(\gamma-1)}}\right)\right] \frac{\hat{d}(\kappa, 0)}{\left(1+S_{0} t\right)^{(3 / 2) \gamma}} \\
& +\frac{\pi}{3(\gamma-1)}\left(\frac{c_{0} \kappa}{\left|S_{0}\right|}\right)\left[Y_{\nu}(\lambda) J_{\nu}\left(\frac{\lambda}{\left(1+S_{0} t\right)^{(3 / 2)(\gamma-1)}}\right)\right. \\
& \left.-J_{\nu}(\lambda) Y_{\nu}\left(\frac{\lambda}{\left(1+S_{0} t\right)^{(3 / 2)(\gamma-1)}}\right)\right] \frac{\hat{p}(\kappa, 0)}{\left(1+S_{0} t\right)^{(3 / 2) \gamma}},
\end{aligned}
$$

and

$$
\begin{aligned}
\hat{p}(\kappa, t)= & \frac{\pi}{3(\gamma-1)}\left(\frac{\rho_{0} S_{0}}{\kappa^{2}}\right)\left(\frac{c_{0} \kappa}{\left|S_{0}\right|}\right)^{2}\left[J_{\nu^{\prime}}(\lambda) Y_{\nu^{\prime}}\left(\frac{\lambda}{\left(1+S_{0} t\right)^{(3 / 2)(\gamma-1)}}\right)-Y_{\nu^{\prime}}(\lambda) J_{\nu^{\prime}}\left(\frac{\lambda}{\left(1+S_{0} t\right)^{(3 / 2)(\gamma-1)}}\right)\right] \\
& \times \frac{\hat{d}(\kappa, 0)}{\left(1+S_{0} t\right)^{3 \gamma+(1 / 2)}}+\frac{\pi}{3(\gamma-1)}\left(\frac{c_{0} \kappa}{\left|S_{0}\right|}\right)\left[J_{\nu}(\lambda) Y_{\nu^{\prime}}\left(\frac{\lambda}{\left(1+S_{0} t\right)^{(3 / 2)(\gamma-1)}}\right)\right. \\
& \left.-Y_{\nu}(\lambda) J_{\nu^{\prime}}\left(\frac{\lambda}{\left(1+S_{0} t\right)^{(3 / 2)(\gamma-1)}}\right)\right] \frac{\hat{p}(\kappa, 0)}{\left(1+S_{0} t\right)^{3 \gamma+(1 / 2)}},
\end{aligned}
$$


where $\quad \nu=\left(\gamma-\frac{4}{3}\right) /(\gamma-1), \quad \nu^{\prime}=-1 /(3(\gamma-1)), \quad$ and $\lambda=2 c_{0} \kappa /\left(3(\gamma-1)\left|S_{0}\right|\right)$. As a check, $\gamma$ was set to $5 / 3$, giving $\nu=1 / 2$ and $\nu^{\prime}=-1 / 2$, and the solution was reduced to the form for a monatomic gas given by Eqs. (22) and (23).

The Fourier coefficients of the dilatation and the pressure given in Eqs. (A5) and (A6) for general values of $\gamma$ could be integrated to form statistics of interest as was done for the monatomic case. However, given its complicated nature, the solution is left in the current form.

${ }^{1}$ V. A. Sabelnikov, "Pressure fluctuations generated by uniform distortion of homogeneous turbulence," Fluid Mech.-Sov. Res. 4, 46 (1975).

${ }^{2}$ P. A. Durbin and O. Zeman, "Rapid distortion theory for homogeneous compressed turbulence with application to modelling," J. Fluid Mech. 242, 349 (1992).

${ }^{3}$ L. Jacquin, C. Cambon, and E. Blin, "Turbulence amplification by a shock wave and rapid distortion theory," Phys. Fluids A 5, 2539 (1993).

${ }^{4}$ C. Cambon, G. N. Coleman, and N. N. Mansour, "Rapid distortion analysis and direct simulation of compressible homogeneous turbulence at finite Mach number,', J. Fluid Mech. 257, 641 (1993).

${ }^{5}$ C. M. Bender and S. A. Orszag, Advanced Mathematical Methods for Scientists and Engineers (McGraw-Hill, New York, 1978).

${ }^{6}$ G. N. Coleman and N. N. Mansour, "Modeling the rapid spherical compression of isotropic turbulence,"’ Phys. Fluids A 3, 2255 (1991).

${ }^{7}$ G. N. Coleman and N. N. Mansour, "Simulation and modeling of homogeneous compressible turbulence under isotropic mean compression," in Turbulent Shear Flows 8 (Springer, Berlin, 1992).
${ }^{8}$ G. A. Blaisdell, N. N. Mansour, and W. C. Reynolds, "Numerical simulations of compressible homogeneous turbulence,' Report TF-50, Department of Mechanical Engineering, Stanford University, 1991.

${ }^{9}$ J. C. R. Hunt and D. J. Carruthers, "Rapid distortion theory and the 'problems' of turbulence,', J. Fluid Mech. 212, 497 (1990).

${ }^{10}$ R. S. Rogallo, "Numerical experiments in homogeneous turbulence," NASA Technical Memorandum 81315, 1981.

${ }^{11} \mathrm{O}$. Zeman, "Dilatational dissipation: The concept and application in modeling compressible mixing layers," Phys. Fluids A 2, 178 (1990).

${ }^{12} \mathrm{O}$. Zeman, "'On the decay of compressible isotropic turbulence," Phys. Fluids A 3, 951 (1991).

${ }^{13}$ S. Sarkar, G. Erlebacher, and M. Y. Hussaini, "The analysis and modeling of dilatational terms in compressible turbulence,' J. Fluid Mech. 227, 473 (1991).

${ }^{14}$ S. Sarkar, "The pressure-dilatation correlation in compressible flows," Phys. Fluids A 4, 2674 (1992).

${ }^{15} \mathrm{R}$. Aris, Vectors, tensors, and the basic equations of fluid mechanics (Prentice-Hall, New Jersey, 1962).

${ }^{16}$ T. M. Apostol, Mathematical Analysis (Addison-Wesley, Reading, 1974).

${ }^{17}$ B. Aupoix, G. A. Blaisdell, W. C. Reynolds, and O. Zeman, "Modeling the turbulent kinetic energy equation for compressible, homogeneous turbulence," in Proceedings of the 1990 Summer Program (NASA Ames/ Stanford Center for Turbulence Research, 1990), pp. 63-74.

${ }^{18}$ G. P. Zank and W. M. Matthaeus, "The equations of nearly incompressible fluids. I. Hydrodynamics, turbulence, and waves," Phys. Fluids A 3, 69 (1991).

${ }^{19}$ M. Abramowitz and I. A. Stegun, Handbook of Mathematical Functions (Dover, New York, 1972). 